\title{
CONSISTENCY ASPECTS OF OUT-OF-PLANE BENDING, TORSION AND SHEAR IN A QUADRATIC CURVED BEAMM ELEMENT
}

\author{
B. P. NAGANARAYANA AND G. PRATHAP
}

Structural Sciences Division, National Aeronautical Laboratory, Bangalore, India 560017

\section{SUMMARY}

Curved beams in civil engineering applications call for out-of-plane bending and torsion under the action of out-of-plane transverse shear loads. The design of a quadratic displacement curved beam element capable of representing shear deformation as in the Timoshenko beam theory requires special attention to the manner in which the shear strain is represented. Field-inconsistent representations of the out-of-plane transverse shear strain will result in a loss of efficiency and introduce spurious oscillations in the bending moment, torsional moment and shear force. The optimal field-consistent assumed strain interpolation for shear is derived and it is demonstrated to posses very high accuracy which is free from spurious force and moment oscillations.

\section{INTRODUCTION}

Most studies ${ }^{1-4}$ on the formulation of curved beam elements relate to in-plane bending and describe mainly the field-consistency requirements for membrane strains and shear strains and how membrane and shear locking and various combinations of violent stress oscillations originate if these conditions are violated. The application of the consistency paradigm to redesign the assumed strain fields correctly such that these deficiencies vanish is straightforward.

In this paper, we study the extension of these concepts to the consistency requirements called for when the quadratic curved beam element is designed to be applied in situations where it must undergo out-of-plane bending and torsion under the action of shear forces. The curved beam element chosen for study here has three nodes, allowing a quadratic isoparametric representation and includes shear deformation according to the Timoshenko beam theory. Although the exactly integrated form of this element does not have severe locking problems, it does have a loss of accuracy and spurious force and moment oscillations if the transverse shear strain field is not modelled in a consistent fashion. The issues involved in the formulation of this quadratic curved beam element are critically examined to show that two consistency conditions must be assured in describing the constrained out-of-plane transverse shear strain field and the term describing the curved centroidal axis appearing in the denominator of all the strain terms.

\section{DESCRIPTION OF ELEMENT GEOMETRY}

Geometry

Figure 1 shows the geometry of the general beam element. In order to describe the out-of-plane behaviour clearly, it is necessary to distinguish four sets of co-ordinate systems. The global 0029-5981/90/110431-13\$06.50

C) 1990 by John Wiley \& Sons, Ltd. 


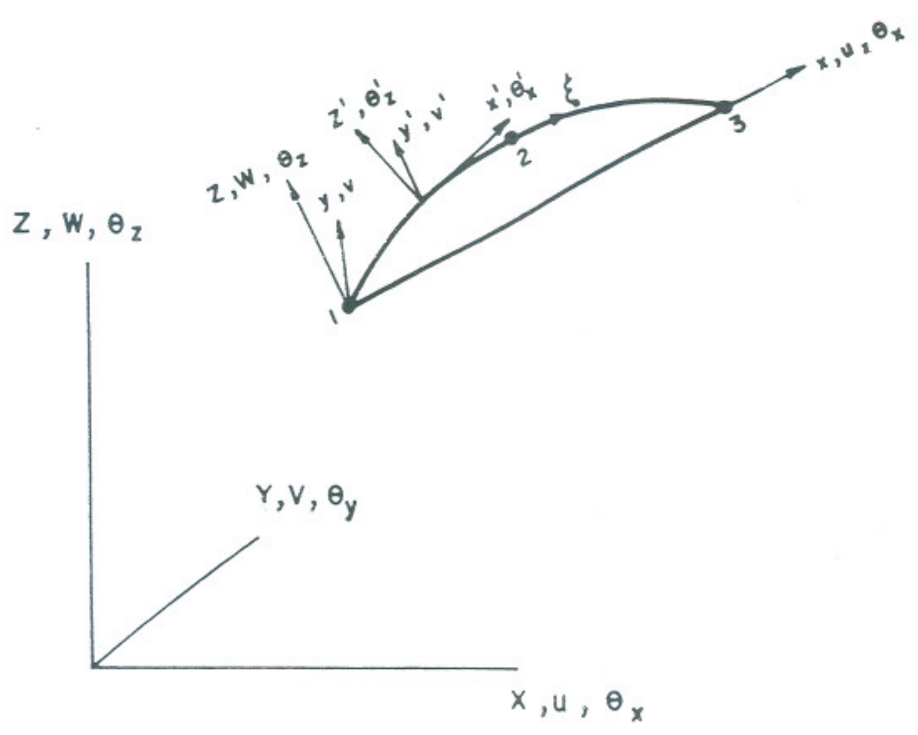

Figure 1. Geometry of the general curved beam element

Cartesian system $\left(X, Y, Z\right.$ and degrees of freedom $\left.U, V, W, \theta_{x}, \theta_{y}, \theta_{z}\right)$ is the principal one in which the nodal co-ordinates and the global deformation are described. The element strains have to be determined in a local running Cartesian co-ordinate system $\left(x^{\prime}, y^{\prime}, z^{\prime}\right.$ and degrees of freedom $v^{\prime}, \theta_{x}^{\prime}$, $\left.\theta_{z}^{\prime}\right)$ in which $x^{\prime}$ is assumed to be both normal to the element cross-section and tangent to the element centroidal axis, the plane $x^{\prime}-z^{\prime}$ describes the plane of curvature, and $y^{\prime}$ is orthogonal to $x^{\prime}-z^{\prime}$ and is the direction of the out-of-plane action. It is assumed for simplicity of illustrating the consistency concepts that the $y^{\prime}$ - and $z^{\prime}$-axes coincide with the principal axes of inertia. It is also necessary to introduce an element plane fixed Cartesian co-ordinate system $(x, y, z)$ as shown in Figure 1 so that the curvature transformation terms can be projected between the $(x, y, z)$ and $\left(x^{\prime}, y^{\prime}, z^{\prime}\right)$ systems initially, as this gives a better insight into how the consistency requirements of the crucial strain terms emerge. The fourth system is the natural curvilinear parameter $\xi$ running along the element centroidal axis, and the isoparametric description is made in terms of this coordinate.

\section{Global to element plane transformation}

The nodal co-ordinates are input in the global Cartesian system $(X, Y, Z)$. The element plane fixed Cartesian system $(x, y, z)$ is oriented as shown in Figure 1 so that the $x$-axis lies through the nodes 1 and 3 and the $x-z$ plane is fixed so that all three nodes lie on this plane. The $y$-axis will be orthogonal to the $x-z$ plane. It is very simple now to determine the orthogonal transformation matrix from the $(X, Y, Z)$ system to the $(x, y, z)$ system and vice versa, and also to determine the co-ordinates $\left(x_{1}, z_{1}\right),\left(x_{2}, z_{2}\right)$ and $\left(x_{3}, z_{3}\right)$ of the three nodes in the element plane system. For further simplicity, it is assumed that the node 2 is exactly midway along the centroidal axis between the two end nodes so that $x_{2}=0.5\left(x_{1}+x_{3}\right)$ and also that $z_{1}=z_{3}=0$. This permits a very simple description of the element geometry and considerably simplifies our study of the role the curved geometry plays in the consistency aspects.

If $\mu_{i}$ represents the direction cosine vectors in the three orthogonal directions shown in Figure 2, the direction cosine matrix is

$$
[\mu]=\left[\begin{array}{lll}
\mu_{1} & \mu_{2} & \mu_{3}
\end{array}\right]
$$


so that the transformation from global to element plane fixed system is

$$
\left\{\begin{array}{c}
x \\
y \\
z
\end{array}\right\}=[\mu]^{-1}\left\{\begin{array}{c}
X \\
Y \\
Z
\end{array}\right\} \quad\left\{\begin{array}{c}
X \\
Y \\
Z
\end{array}\right\}=[\mu]\left\{\begin{array}{c}
x \\
y \\
z
\end{array}\right\}
$$

Element plane fixed to running local system

The transformation between the running local Cartesian system and the element plane fixed Cartesian system is derived from the fact that $x^{\prime}$ is normal to the element cross-section and also tangential to the curved centroidal axis and that $y^{\prime}$ is coincident with $y$. Thus, if $\lambda_{i}$ are the direction cosine vectors shown in Figure 2, we can easily show that

$$
\begin{aligned}
& \left\{\lambda_{1}\right\}=\frac{1}{\sqrt{D}}\left\{\begin{array}{c}
x_{, \xi} \\
0 \\
z_{, \xi}
\end{array}\right\} \\
& \left\{\lambda_{2}\right\}=\left\{\mu_{2}\right\} \\
& \left\{\lambda_{3}\right\}=\frac{1}{\sqrt{D}}\left\{\begin{array}{c}
-z_{, \xi} \\
0 \\
x_{, \xi}
\end{array}\right\}
\end{aligned}
$$

where $D=\left(x_{, \xi}^{2}+z_{, \xi}^{2}\right)$.

\section{DESCRIPTION OF ELEMENT STRAIN FIELDS}

For a curved beam designed to represent out-of-plane action, three strain fields have to be taken into account:

out-of-plane flexure

$$
\chi^{\prime}=\theta_{z, x^{\prime}}^{\prime}
$$

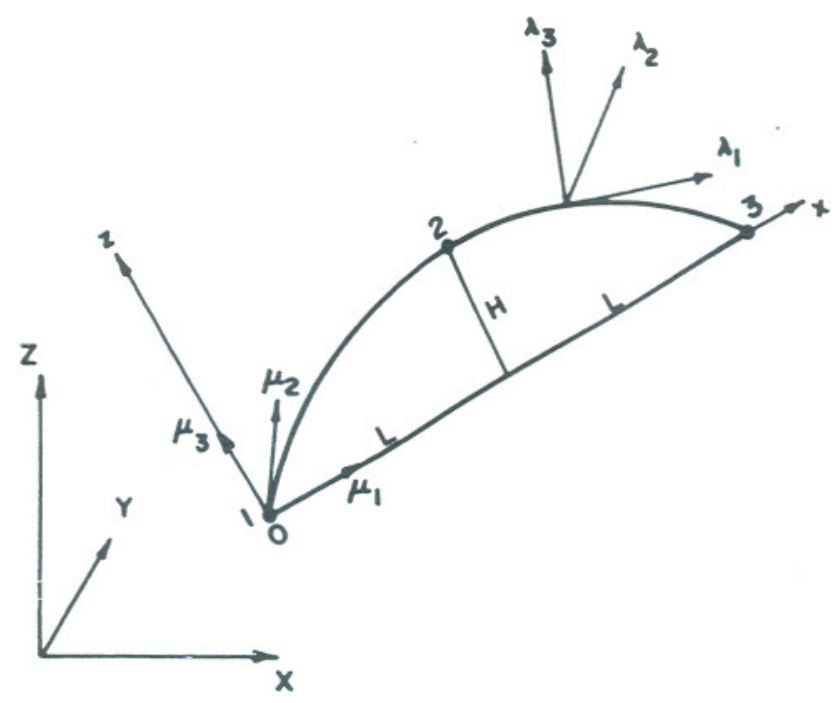

Figure 2. Orthogonal transformation schemes from global Cartesian to element plane Cartesian $(\mu)$ and from element plane Cartesian to local running Cartesian $(\lambda)$ 
$\begin{array}{ll}\text { torsion } & \omega^{\prime}=\theta_{x, x^{\prime}}^{\prime} \\ \text { out-of-plane transverse shear } & \gamma^{\prime}=\theta_{z}^{\prime}-v_{, x^{\prime}}^{\prime}\end{array}$

The displacement fields are assumed to be internolated using a quadratic representation described for further convenience in terms of Legendre polynomials as

$$
\begin{gathered}
v=a_{0}+a_{1} \xi-a_{2} / 3\left(1-\xi^{2}\right) \\
\theta_{x}=b_{0}+b_{1} \xi-b_{2} / 3\left(1-3 \xi^{2}\right) \\
\theta_{z}=c_{0}+c_{1} \xi-c_{2} / 3\left(1-3 \xi^{2}\right)
\end{gathered}
$$

where coefficients such as $a_{i}, b_{i}$ and $c_{i}$ can be related to nodal values of $v, \theta_{x}$ and $\theta_{z}$ as $a_{0}=$ $\left(v_{1}+4 v_{2}+v_{3}\right) / 6, a_{1}=\left(v_{3}-v_{1}\right) / 2$ and $a_{2}=\left(v_{1}-2 v_{2}+v_{3}\right) / 2$, etc. Using these displacement fields in (3a) to (3c) we get the three strain fields expanded in the Legendre polynomial form as follows.

Out-of-plane bending strain

$$
\chi^{\prime}=\frac{1}{D}\left[2 H \xi\left(b_{1}+2 b_{2} \xi\right)+L\left(c_{1}+2 c_{2} \xi\right)\right]
$$

Torsional strain

$$
\omega^{\prime}=\frac{1}{D}\left[L\left(b_{1}+2 b_{2} \xi\right)-2 H \xi\left(c_{1}+2 c_{2} \xi\right)\right]
$$

Out-of-plane transverse shear strain

$$
\begin{aligned}
\gamma^{\prime}= & \frac{1}{\sqrt{D}}\left(-v_{, \xi}+2 H \xi \theta_{x}+L \theta_{z}\right) \\
= & \frac{1}{\sqrt{D}}\left\{\left[-a_{1}+2 H / 3 b_{1}+L c_{0}\right]\right. \\
& +\xi\left[-2 a_{2}+2 H\left(b_{0}+4 / 15 b_{2}\right)+L c_{1}\right] \\
& -1 / 3\left(1-3 \xi^{2}\right)\left[2 H b_{1}+L c_{2}\right] \\
& \left.-1 / 5\left(3 \xi-5 \xi^{3}\right)\left[2 H b_{2} / 2\right]\right\}
\end{aligned}
$$

Of the above strain fields, the transverse shear is the only constrained one requiring the corresponding strain energy to vanish in the 'thin' plate regimes. Hence, it is this strain field which needs the 'field-consistency' requirements to be met.

\section{FIELD-CONSISTENCY ASPECTS}

We shall proceed now to a study of the consistency requirements demanded by the shear strain field described above to determine how an assumed strain field interpolation can be set up in an optimally consistent way and also examine the performance if it includes various degrees of inconsistency.

In the expression for the transverse shear strain field in equation (7), consistency is now sought between terms such as $v_{, \xi}, H \xi \theta_{x}$ and $L \theta_{z}$, and these are expanded carefully in terms of the Legendre polynomials as shown. It should be noted here that, since the Legendre polynomial is specially designed to be 'orthogonal' in the domain of Gaussian quadrature, the coefficients of the 
Legendre polynomials directly emerge as the constraints:

$$
\begin{aligned}
-a_{1}+2 H / 3 b_{1}+L c_{0} & \rightarrow 0 \\
-2 a_{2}+2 H\left(b_{0}+4 / 15 b_{2}\right)+L c_{1} & \rightarrow 0 \\
2 H b_{1}+L c_{2} & \rightarrow 0 \\
2 H b_{2} & \rightarrow 0
\end{aligned}
$$

in the 'thin' plate limits (since the associated shear strain energy is constrained to vanish). For a straight beam, with $H=0$, there will be only three constraints and the third constraint as seen in equation (8c) is of the identical form seen for the in-plane transverse shear term in a straight quadratic beam element. For a curved beam, the out-of-plane shear strain consistency terms include two spurious constraints, represented by equations $(8 \mathrm{c})$ and $(8 \mathrm{~d})$.

Now, the correct way of deriving the field-consistent shear strain field is to drop the 'inconsistent' Legendre terms. This procedure automatically satisfies the orthogonality or variational correctness condition discovered on seeking the equivalence of this assumed strain approach to the minimum total potential energy principle to the mixed variational approach using principles like Hellinger-Reissner or $\mathrm{Hu}$-Washizu theorems. ${ }^{5}$ We shall therefore examine the three possible variations that can be studied:

$\mathrm{LV}=0$, in which all inconsistencies are retained;

$\mathrm{LV}=1$, in which the two true constraints and the quadratic inconsistency is retained; and

$\mathrm{LV}=2$, with the assumed out-of-plane transverse shear strain having only the true constraints and correct to a linear interpolation.

In fact, $\mathrm{LV}=2$ represents the variationally correct and field-consistent version of the element.

\section{Consistency of definition of $D$}

We see from the various strain definitions such as equation (7) for the shear strain and equations (5) and (6) above for the flexural and torsional strains that the geometry term $D$ or $\sqrt{D}$ appears in the denominator. It therefore plays a contributory role to the discretized strain interpolation. We can illustrate the idea by examining the case of the shear strain in equation (7). Note that, when the numerator is made field-consistent (i.e. $L V=2$ ), it can sense only a linear interpolation of the terms derived from the nodal degrees of freedom. However, $D$ in the denominator defines a quadratic variation in $\xi$. Thus, a conflict arises whereby strains are derived from the nodal degrees of freedom up to a linear accuracy and these are modified by a quadratically varying term representing geometry. We shall see later that it is more reasonable to restrict $D$ also to linear accuracy when the constrained strain fields are being computed. We find it convenient to use in this instance

$$
D^{\prime}=L^{2}+4 H^{2} / 3
$$

by noting that $D=\left(L^{2}+4 H^{2} / 3\right)-4 H^{2} / 3\left(1-3 \xi^{2}\right)$ and arguing that a representation up to linear accuracy is obtained by omitting the quadratic Legendre polynomial and the term associated with it. To examine these distinctions clearly in the section on numerical experiments later, we shall consider two levels of geometry description:

LG $=0$ where $D$ is used; and

LG $=1$ where $D^{\prime}$ is used for all strain fields. 


\section{ELEMENT STIFFNESS MATRICES AT VARIOUS LEVELS}

The constitutive equations relating the generalized stresses, shear force $Q$, torsion moment $T$ and bending moment $M$ to the strains described above can be written in terms of the cross-sectional properties and the material constants. These details and the general derivation of the element stiffness matrices are omitted here as they are well understood. ${ }^{5}$

We shall, however, examine carefully the various levels of assumed shear strain field and also the correct order of numerical integration to be used to ensure that these various levels are exactiy integrated. We use the numbering system CB3.vg to represent the element with assumed strain options corresponding to the use of versions LV, to ensure consistency of the constrained shear strain field and the use of option LG to maintain a 'linear' interpolation form for $D$ in the denominator of the strain fields to understand the importance of using a geometry representation that is compatible with the strain approximations. Thus, the principal forms that need very close investigation are

element CB3.21 is the optimal element (with $L V=2$ and $L G=1$ ) as we shall see later, elements $\mathrm{CB} 3.11$ and $\mathrm{CB} 3.01$ shows how this element behaves if the transverse shear strains are inconsistent $(\mathrm{LV}=1,0$ and $\mathrm{LG}=1)$,

element CB3.20 shows the differences if geometry representation for $D$ is not compatible with the strain definitions $(\mathrm{LV}=2$ and $\mathrm{LG}=0)$.

We see that, for option $L V=0$, the shear strain field has a description up to cubic order and a 4-point Gaussian integration is mandatory to integrate this energy term completely. We therefore adopt a 3-point rule for all other energy terms and a 4-point rule for the transverse shear strain energy.

\section{A PRIORI QUALITATIVE ERROR PROJECTIONS}

Earlier studies ${ }^{1-5,7-9}$ have shown that in many cases it is possible to proceed from the consistency paradigm with an operational procedure described as the functional re-constitution process to obtain useful estimates for the manner in which locking will take place, errors will propagate as the penalty multipliers increase and how various strain/stress oscillations are induced and how their severity can be related to the penalty parameters etc. However, in this problem, we find that the parameters describing the strain interpolations are so intricately coupled that it is not easy to isolate the influences of each inconsistency and use it to determine in a precise quantitative way, the additional stiffening ${ }^{10}$ involved. However, it will still be worthwhile to identify in a qualitative way how the inconsistencies disturb the unconstrained strain fields and cause poorer preformance and stress oscillations in the CB3.11 and the CB3.01 elements.

To see the inter-relationships more clearly, it will be useful to expand the strain fields in terms of the Legendre polynomial as follows

$$
\begin{aligned}
\chi^{\prime} & =\frac{1}{D}\left\{\left[L c_{1}+4 H / 3 b_{2}\right]+\xi 2\left[H b_{1}+L c_{2}\right]-\left(1-3 \xi^{2}\right)\left[4 H / 3 b_{2}\right]\right\} \\
\omega^{\prime} & =\frac{1}{D}\left\{\left[L b-4 H / 3 c_{2}\right]+\xi 2\left[L b_{2}-H c_{1}\right]-\left(1-3 \xi^{2}\right)\left[4 H / 3 c_{2}\right]\right\}
\end{aligned}
$$

From equations (7) and (8c), we observe that the spurious constraint generated by the shear strain field at the quadratic level involves the constants $b_{1}$ and $c_{2}$. It is this inconsistency that initiates 
the quadratic oscillation in the shear strain predictions from a quadratic beam element. ${ }^{3,4,9} \mathrm{We}$ are now interested to see if any additional features appear due to this. It is seen in equation (9a) that the linearly varying part of the bending strain field is determined by these same constants. Thus, we can argue that, in an inconsistent formulation (i.e. in the CB3.11 element), the disturbance in the constants $b_{1}$ and $c_{2}$ will be reflected as an additional spurious linear oscillation. Proceeding next to the torsional strain field in equation (9b), we notice that the constants $b_{1}$ and $c_{2}$ appear in both the constant part and the quadratic part. Thus, there will be errors in both the representation of the constant part of the torsional strain field and an additional spurious quadratic oscillation.

The CB3.01 element has a cubic inconsistency as well, but this will play a very insignificant role compared to the inconsistency already excited at the quadratic level and therefore this element will produce results that are very nearly identical to that produced by the CB3.11 element.

\section{NUMERICAL EXPERIMENTS}

We shall investigate only those versions of the element needed to confirm the predictions we have made in the previous section. To bring out these aspects lucidly it is necessary to construct suitable example problems such as the 'nearly straight' cantilever beam under torsion and the curved beam under shear force. The convergence of displacements and rotations when the CB3.21

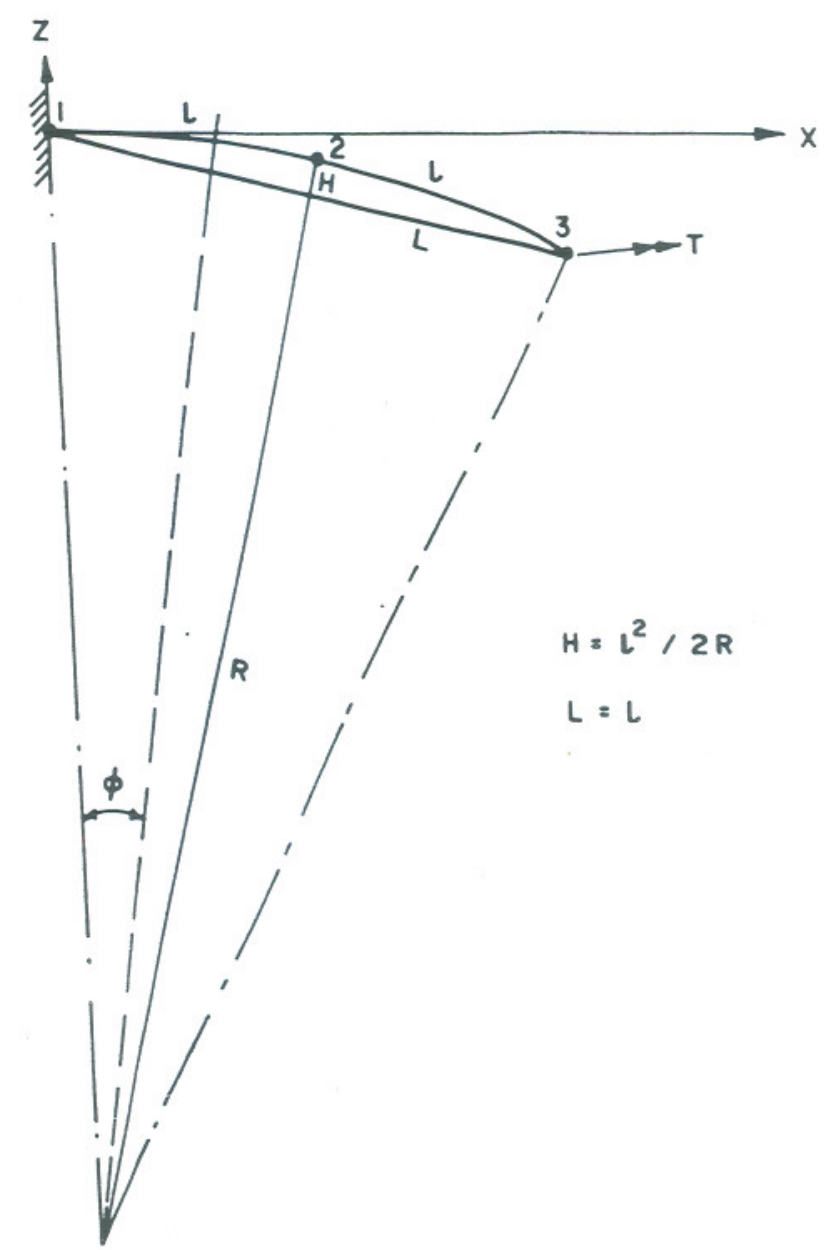

Figure 3. Geometry of nearly straight cantilever beam 
elements were used was extremely good and will not be/reported here. The CB3.11 and CB3.01 elements yielded less accurate displacement and rotation predictions and these can be attributed to the lack of consistency in the shear strain definitions. We shall therefore concentrate attention on the stress predictions from the various element versions.

Single element models of 'nearly straight' cantilever under tip torsional moment

A beam of length $2 L=100$ is given a small curvature corresponding to a radius $R=1000$ (see Figure 3). A torsional moment $T=1$ is applied at the tip as shown. The elastic and section properties chosen are $E=10^{7}, G=0.5 \times 10^{7}, I=0.083333$ and $J=0.166667$. The entire beam is modelled using a single 3 -node element.

From the statics of equilibrium, one can obtain an estimate for the bending moment $M_{z}^{\prime}$ and the torsional moment $M_{x}^{\prime}$ at a cross-section $\xi$ for the single element model of the cantilever as

$$
\begin{aligned}
& M_{z}^{\prime}=\sin [(1+\xi) / 20] \\
& M_{x}^{\prime}=\cos [(1+\xi) / 20]
\end{aligned}
$$

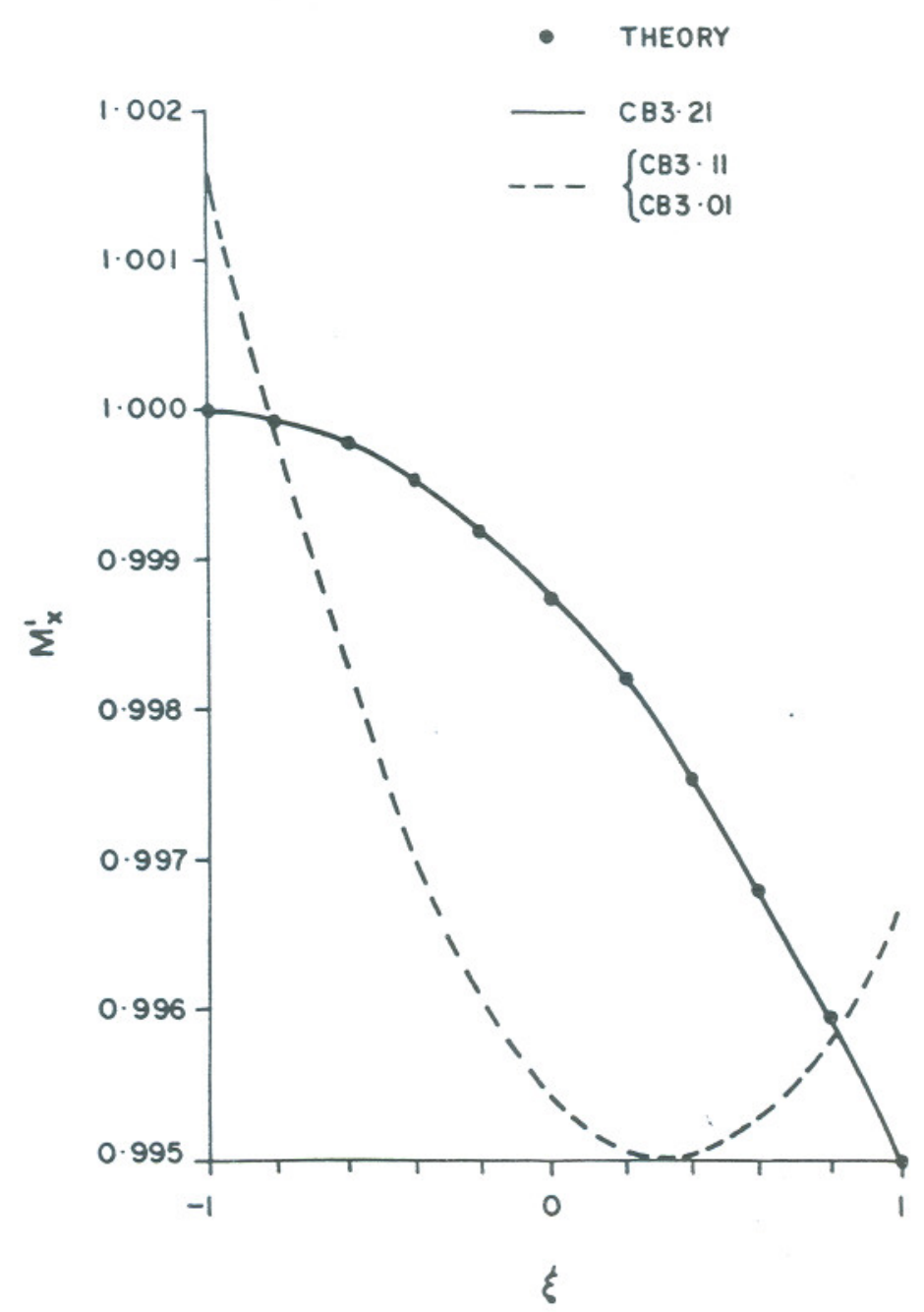

Figure 4. Nearly straight beam under torsion-torsional moments along beam 
Figure 4 shows the torsional moments computed from equation (10b) and compares it with the predictions from the CB3.21, CB3.11 and CB3.01 idealizations. It is seen that the CB3.21 element gives practically the exact answers. However, the two inconsistent elements gave answers which are considerably at variance from the correct answers. A very close examination of the results revealed that the inconsistency in the shear strain field excited disturbances in the constant part of the torsional strain field and introduced an additional spurious quadratic oscillation so that the differences can be described for the results shown on Figure 4 as

$$
M_{x}^{\prime}(\mathrm{CB} 3.11)=M_{x}^{\prime}(\mathrm{CB} 3.21)-1 / 600-1 / 600\left(1-3 \xi^{2}\right)
$$

These changes are exactly as we had anticipated earlier in our error projections.

Figure 5 shows the out-of-plane bending moments $M_{z}^{\prime}$. Again, the CB3.21 model performs correctly. The field-inconsistent versions show a large linear oscillation that completely reverses the correct trend of bending moment distribution. These observations again confirm the predictions made earlier.

Figure 6 describes the quadratic oscillations in the shear force obtained from the CB3.11 and the CB3.01 models. The additional cubic oscillations expected in the latter model are too insignificant to be represented here, showing that the predominant inconsistency is the quadratic one.

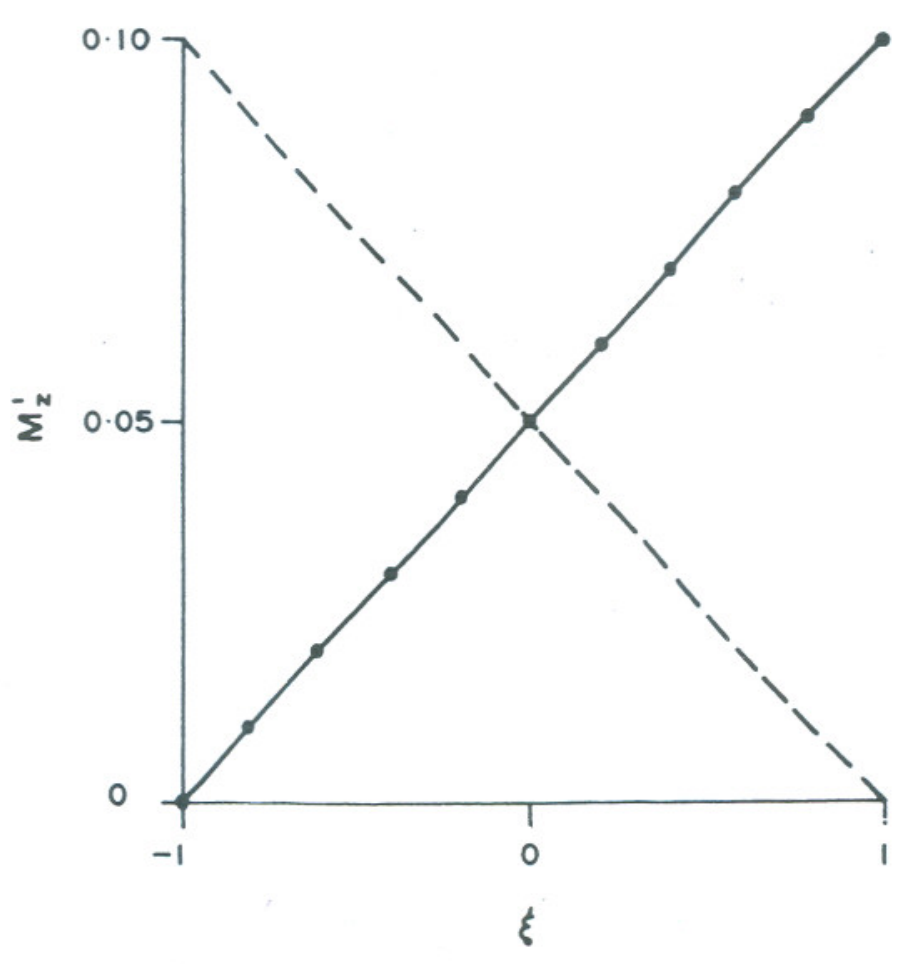

Figure 5. Nearly straight beam under torsion-out-of-plane bending moments along beam 
- THEORY

$-\mathrm{CB3} \cdot 21$

$--\left\{\begin{array}{l}\text { Ce3. } 11 \\ \text { CB3. } 91\end{array}\right.$

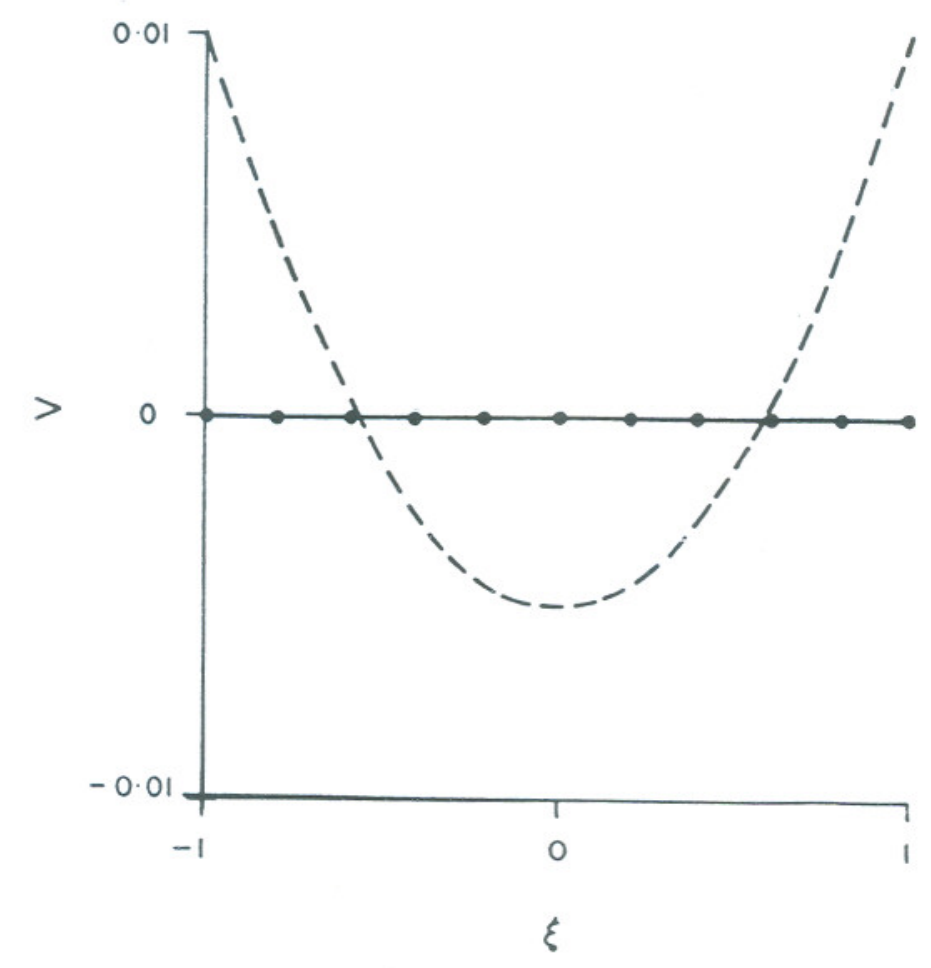

Figure 6. Nearly straight beam under torsion-shear force along beam

\section{Curved beam under shear force}

Figure 7 shows a curved beam forming the quadrant of a circle. The radius of arc of the circle is 5.0 and the elastic and section properties remain exactly as for the example above. An out-ofplane transverse shear force $P$ is applied at the free end.

Figure 8 shows the bending moments obtained from a one and two element idealization of the curved beam. The convergence is very good for the CB3.21 models. Also shown is the poor

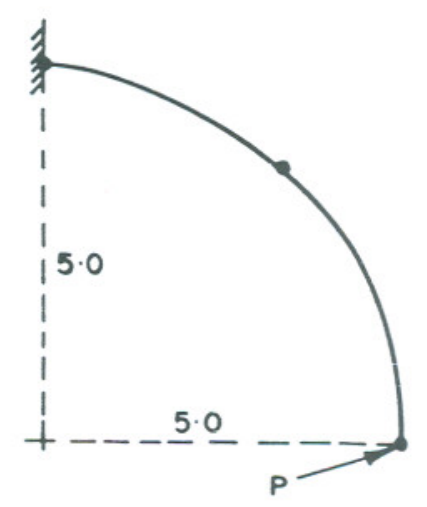

Figure 7. Curved cantilever beam with out-of-plane transverse shear force 


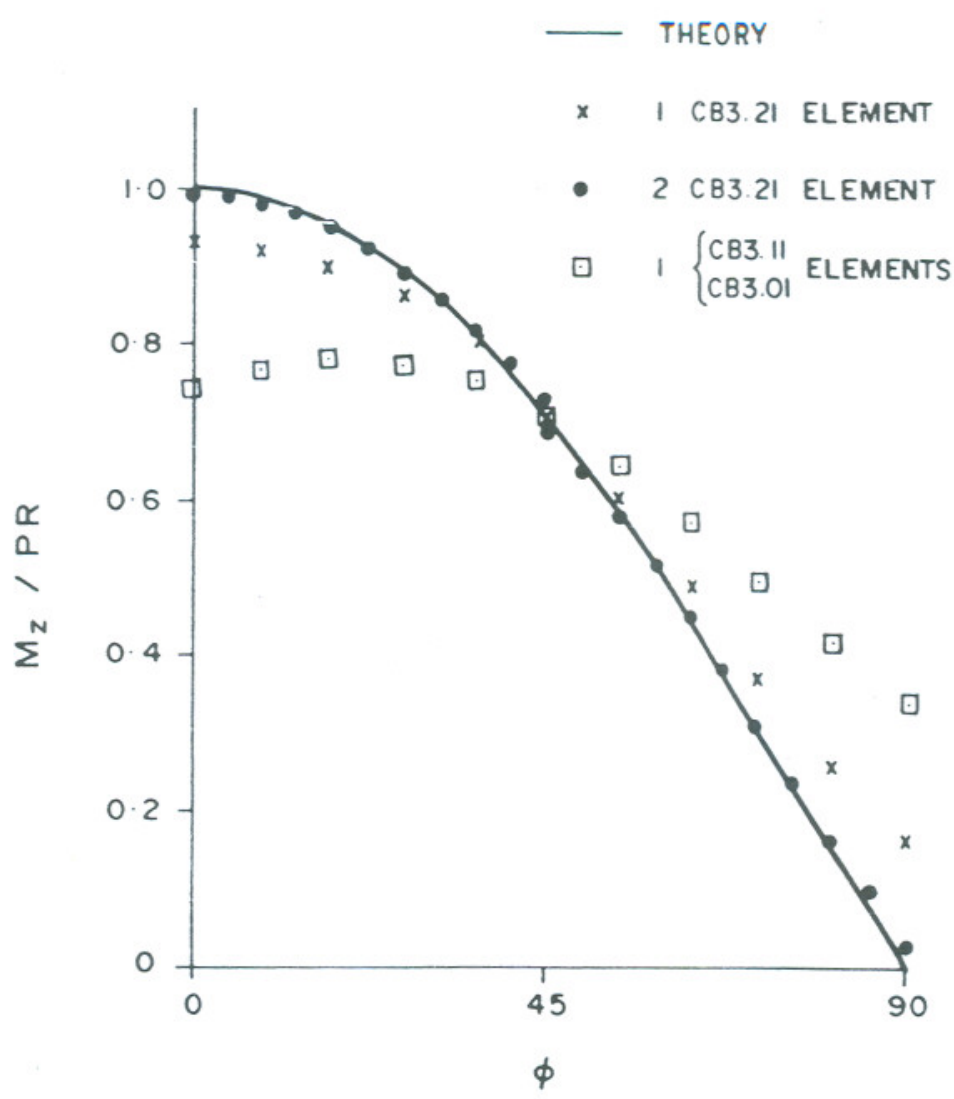

Figure 8. Curved cantilever beam under out-of-plane transverse shear load-bending moment

behaviour seen with the inconsistent element. There is a noticeable shift in the predicted bending moments due to the disturbance in the linear component.

Figure 9 shows the predictions of torsional moment. The CB3.21 models converge rapidly to the correct answers. In this case, the inconsistent elements also yield correct answers (not shown here).

Figure 10 demonstrates the importance of introducing a consistent representation of $D$ as far the denominator of the strain fields are concerned. The shear forces from a CB3.21 and a CB3.20 model show that the former recovers the constant value of shear force correctly while the latter introduces a 'quadratic' type variation that can be traced to the $D$ term in the strain field. Thus, it is more appropriate to use the smoothed representation of $D$ to interpret the strains derived from the nodal displacements and rotations when the stresses are reported. Also shown for completeness are the actual quadratic shear force oscillations triggered off by the field-inconsistency in the CB3.11 and the CB3.01 elements.

\section{CONCLUSIONS}

In this paper, we have examined the consistency requirements demanded by the out-of-plane transverse shear strain definition for a curved beam bending and twisting under out-of-plane loads. The force oscillations that result if inconsistencies are not removed are predicted and are confirmed using numerical experimentation. The optimal form of representation of the shear strain field is derived and is shown to have superior accuracy and convergence in its ability to recover the correct force fields. 


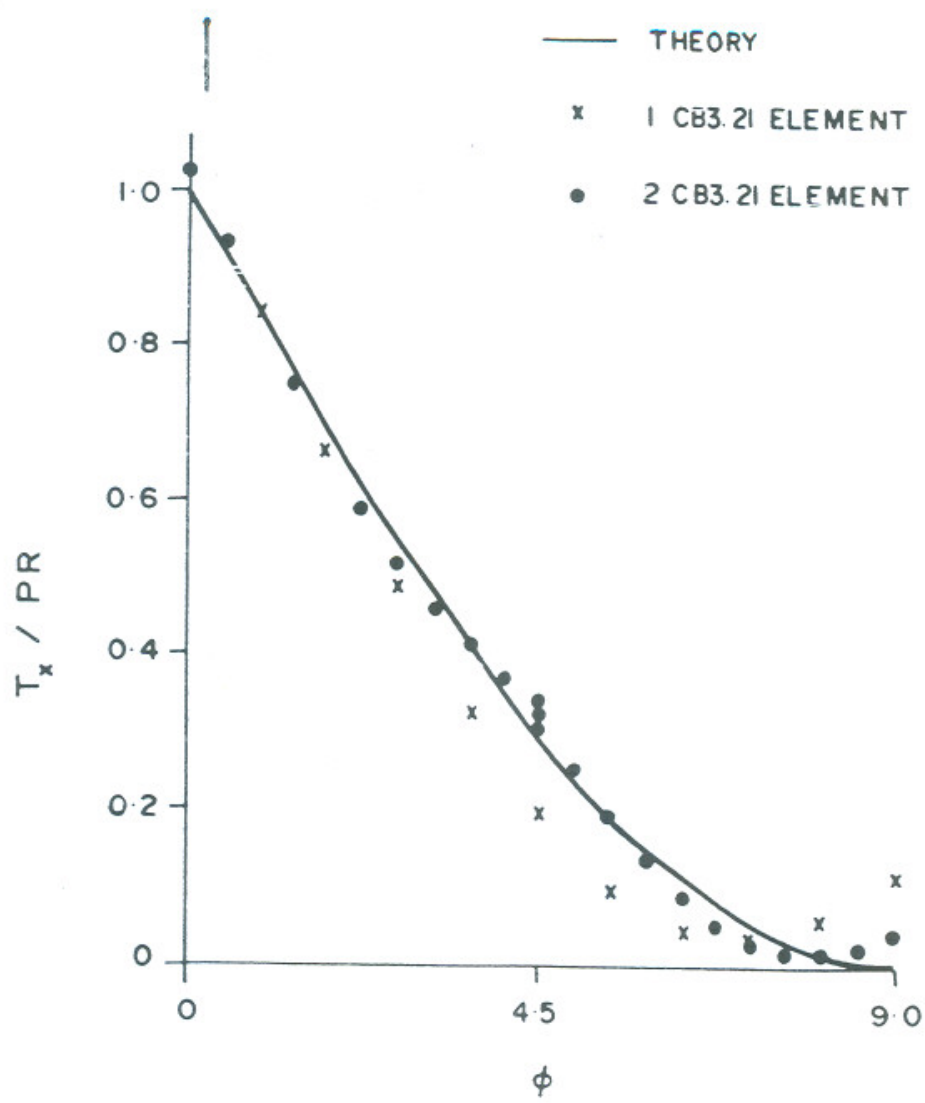

Figure 9. Curved cantilever beam under out-of-plane transverse shear load-torsional moment

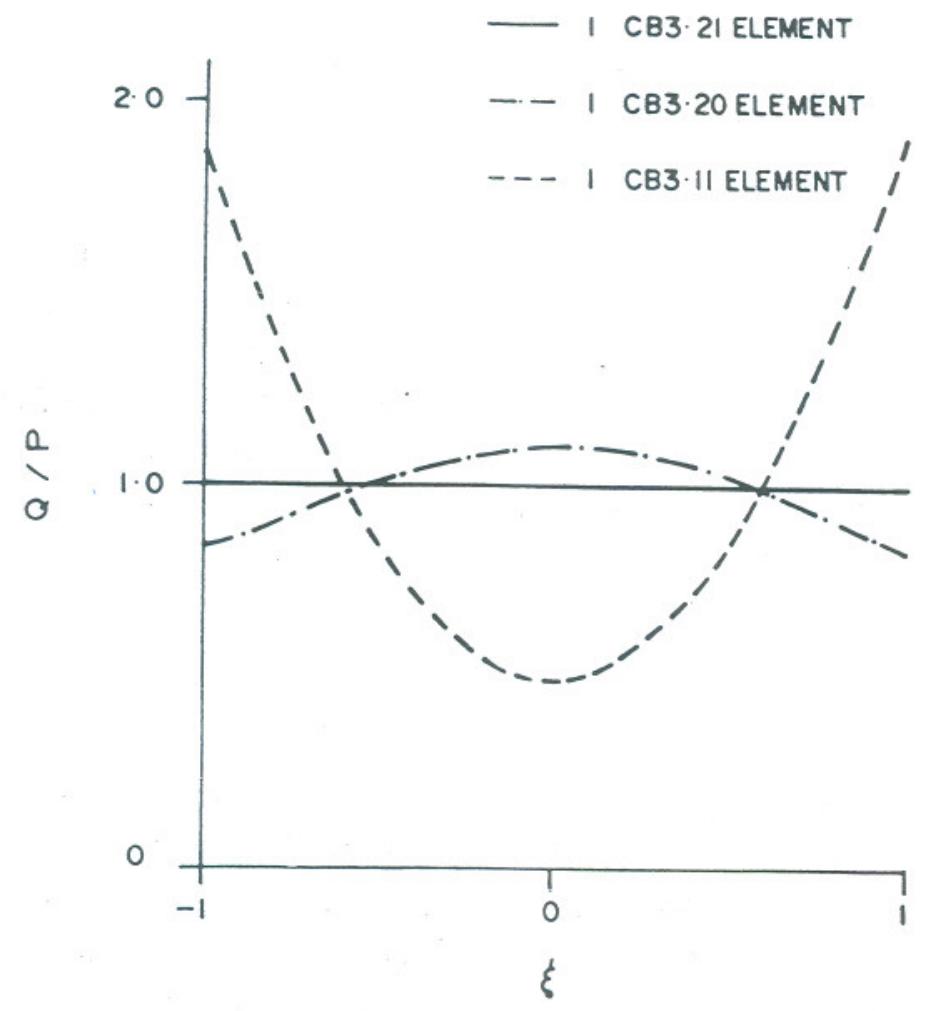

Figure 10. Curved cantilever beam under out-of-plane transverse shear load-shear forces 


\section{ACKNOWLEDGEMENTS}

The authors gratefully acknowledge the constant encouragement and support received from Professor R. Narasimha, Director, National Aeronautical Laboratory and Dr K. N. Raju, Head, Structures Division.

\section{REFERENCES}

1. G. Prathap, 'The curved beam/deep arch/finite ring element re-visited', Int. j. numer. methods eng., 21, 389-407 (1985).

2. C. Ramesh Babu and G. Prathap, 'A linear thick curved beam element', Int. j. numer. methods eng., 23, 1313-1328 (1986).

3. G. Prathap and C. Ramesh Babu, 'An isoparametric quadratic thick curved beam element', Int. j. numer. methods eng., 23, 1583-1600 (1986).

4. G. Prathap and C. Ramesh Babu, 'Field-consistency and violent stress oscillations in the finite element method', Int.j. numer. methods eng., 24, 2017-2033 (1987).

5. G. Prathap and B. P. Naganarayana, 'Analysis of locking and stress oscillations in a general curved beam element', Int. j. numer. methods eng., 30, 177-200 (1990).

6. J. Jirousek, 'A family of variable section curved beam and thick shell or membrane stiffening isoparametric elements', Int. j. numer. methods eng., 17, 171-186 (1981).

7. G. Prathap, 'Field-consistency-Toward a science of constrained multi-strain-field finite element formulations', SADHANA, 9, 345-353 (1986).

8. G. Prathap and G. R. Bhashyam, 'Reduced integration and the shear flexible beam element', Int. j. numer. methods eng., 18, 195-210 (1982).

9. G. Prathap and C. Ramesh Babu, 'Field-consistent strain interpolations for the quadratic shear flexible beam element', Int. j. numer. methods eng., 23, 1973-1984 (1986).

10. G. Prathap, 'An additional stiffening parameter measure of error of the second kind in the finite element method', Int. j. numer. methods eng., 21, 1001-1012 (1985). 